\title{
Circuit
}

Musiques contemporaines

\section{Jean Mozart Lesage, ou une boussole pour dériver Jean Mozart Lesage, ou une boussole pour dériver}

\section{Michel Gonneville}

Volume 18, numéro 2, 2008

Postiches et mélanges

URI : https://id.erudit.org/iderudit/018657ar

DOI : https://doi.org/10.7202/018657ar

Aller au sommaire du numéro

Éditeur(s)

Les Presses de l'Université de Montréal

ISSN

1183-1693 (imprimé)

1488-9692 (numérique)

Découvrir la revue

Citer cet article

Gonneville, M. (2008). Jean Mozart Lesage, ou une boussole pour dériver. Circuit, 18(2), 93-114. https://doi.org/10.7202/018657ar

\section{Résumé de l'article}

Le projet Mozart de Jean Lesage, pour trio, est sans doute l'une des oeuvres les plus claires et les plus ludiques de ce compositeur. En présentant par fragments le texte intégral, mais réorchestré, d'un adagio provenant d'une sonate pour piano de Mozart, Lesage donne déjà à l'auditeur des balises perceptibles. À ces fragments se grefferont des commentaires-dérives inspirés par la matière première de ce même adagio, que ce soit sous son aspect thématique (mélodico-rythmique) ou harmonique, mais aussi parfois selon des directions plus abstraites ou " subliminales ». Au total, avec une grande fantaisie créatrice et une belle liberté, l'oeuvre réalise un équilibre entre accessibilité et sophistication.

À l'aide de tableaux formels et de plusieurs exemples musicaux, l'auteur mène une analyse chronologique de l'oeuvre, émaillée de considérations esthétiques, jusqu'à une conclusion qui commente les propos du compositeur lui-même, et où les notions de baroque et de recontextualisation associées au post-modernisme prennent un sens très concret. 


\section{CAHIER D'ANALYSE}

\section{Jean Mozart Lesage,}

\section{ou une boussole pour dériver}

Michel Gonneville

Pour certains auditeurs, plusieurs œuvres de Jean Lesage apparaissent comme des aventures déroutantes au sein de forêts touffues où les clairières sont rares et les sentiers parfois dissimulés dans la végétation de sous-bois proliférants. Des espèces diverses semblent s'y juxtaposer : fragments de citations possibles, d'allusions à des musiques connues, mais nous marchons trop vite et avons à peine le temps d'identifier, perdus parfois comme dans un rêve.

Cependant, au sein de cette sylviculture, Le projet Mozart apparaît comme une promenade bien balisée qui peut servir d'excellente introduction à la pensée du compositeur, à sa façon de s'intéresser au passé de la musique pour le réinterpréter, pour lui donner un autre sens, pour le réactualiser. La forme en est relativement claire et lisible. Le présent article est le compte rendu d'une analyse, où la consultation de la partition et la référence répétée à l'enregistrement ont permis de ralentir, voire d'arrêter le temps, d'interrompre l'excursion en quelque sorte, afin d'identifier encore plus précisément et en plus grand nombre les éléments qui s'offrent à notre perception pour ensuite mieux saisir les relations qu'ils entretiennent entre eux. Le plaisir des auditions subséquentes ne peut qu'en être accru. Le mot de Rosen (dans Plaisir de jouer, plaisir de penser) semble faible, puisque l'analyse fait plus qu' «éclairer la jouissance»: en faisant «entendre plus », elle l'augmente.

Basée sur l'Andante cantabile ( $2^{\mathrm{e}}$ mouvement) de la Sonate K. 330 en do majeur pour piano de Mozart (auquel Lesage s'était d'ailleurs déjà attaché lors de ses études au conservatoire, à l'occasion d'un travail d'orchestration), l'œuvre a été écrite pour un ensemble classique (violon, violoncelle, piano) à la demande du Trio Fibonacci. Elle se veut, selon les mots du compositeur, une fusion entre le travail de la transcription, de l'interprétation composée et de la composition. 
1. Concernant la numérotation des mesures du mouvement de Mozart, qui est intégralement cité par Lesage et subdivisé pour ce faire en unités de longueur variable, nous avons désigné la première mesure - incomplète comme la mesure o (zéro). Par ailleurs, Lesage ayant identifié dans son analyse du Mozart, et utilisé dans son travail sur ce matériau, des unités phraséologiques qui commencent ou s'achèvent sur des moitiés de mesures, nous avons désigné ces moitiés en ajoutant a ou b au numéro de la mesure pour en désigner respectivement la première et la seconde moitié. Enfin, tout au long de cette analyse nous désignerons les mesures de la partition de Mozart en les faisant précéder d'un $\mathrm{M}$, et celles de la partition de Lesage d'un $\mathrm{L}$.

\section{Forme globale}

D'entrée de jeu, voici un tableau donnant une vue globale de l'œuvre de Lesage, dont nous commenterons ensuite les parties plus en détails'.

Tableau 1. Analyse formelle du projet Mozart de Jean Lesage

\begin{tabular}{|c|c|c|c|c|}
\hline $\begin{array}{l}\text { Mesures } \\
\text { Lesage }\end{array}$ & $\begin{array}{l}\text { Mesures } \\
\text { Mozart }\end{array}$ & $\begin{array}{c}\text { Durée en } \\
\text { croches }\end{array}$ & & Matière harmonique \\
\hline $1-12$ & & 64 & $\begin{array}{l}\text { Introduction sur } \\
\text { itération de mesure Mo }\end{array}$ & \\
\hline $13-19$ & $0-4 a$ & 24 & $\begin{array}{l}\text { Citation de Mozart } \\
\text { réorchestrée } 1\end{array}$ & \\
\hline $20-27$ & & 24 & Commentaire / dérive 1 & \\
\hline $28-32$ & $4 b-8 a$ & 24 & $\begin{array}{l}\text { Citation de Mozart } \\
\text { réorchestrée } 2\end{array}$ & \\
\hline $33-64$ & & 96 & Commentaire / dérive 2 & $f a-d o$ \\
\hline $65-71$ & $8 b-12 a$ & 24 & $\begin{array}{l}\text { Citation de Mozart } \\
\text { réorchestrée } 3\end{array}$ & \\
\hline $72-83$ & & 36 & Commentaire / dérive 3 & Accords symétriques \\
\hline $84-97$ & $12 b-20 a$ & 48 & $\begin{array}{l}\text { Citation de Mozart } \\
\text { réorchestrée } 4\end{array}$ & \\
\hline 98-139 & & 126 & $\begin{array}{l}\text { Commentaire / dérive } 4 \\
\text { en } 3 \text { sections }\end{array}$ & $\begin{array}{l}\text { 1) } d o-s o l \\
\text { 2) } s i b \\
\text { 3) } f a\end{array}$ \\
\hline $140-147$ & $20 b-24 a$ & 24 & $\begin{array}{l}\text { Citation de Mozart } \\
\text { réorchestrée } 5\end{array}$ & \\
\hline $147-157$ & & 60 & Commentaire / dérive 5 & Pédale de $f a$ \\
\hline $157-161$ & $24 b-28 a$ & 24 & $\begin{array}{l}\text { Citation de Mozart } \\
\text { réorchestrée } 6\end{array}$ & \\
\hline $162-210$ & & 142.5 & $\begin{array}{l}\text { Commentaire / dérive } 6 \\
\text { en } 3 \text { sections }\end{array}$ & $\begin{array}{l}\text { 1) réb-do-réb } \\
\text { 2) } f a-r e ́ b-(m i b ?) \\
\text { 3) mib - fa - sol - do- } \\
(I a b)-s i b-r e ́ b-m i b\end{array}$ \\
\hline $211-215$ & $28 b-32 a$ & 24 & $\begin{array}{l}\text { Citation de Mozart } \\
\text { réorchestrée } 7\end{array}$ & \\
\hline $215-230$ & & 90 & $\begin{array}{l}\text { Commentaire / dérive } 7 \text { : } \\
\text { fragmentation }\end{array}$ & \\
\hline $230-234$ & $32 b-36 a$ & 24 & $\begin{array}{l}\text { Citation de Mozart } \\
\text { réorchestrée } 8\end{array}$ & \\
\hline $235-266$ & & 117 & $\begin{array}{l}\text { Commentaire / dérive } 8 \\
\text { en } 5 \text { séquences }\end{array}$ & $\begin{array}{l}\text { 1) lab 2) sib } \\
\text { 3) réb (lab) } \\
\text { 4) do 5) fa }\end{array}$ \\
\hline $267-270$ & $36 b-38 a$ & 12 & $\begin{array}{l}\text { Citation de Mozart } \\
\text { réorchestrée } 9\end{array}$ & \\
\hline $271-280$ & & 30 & Commentaire / dérive 9 & Autour de $f a$ pédale \\
\hline $281-337$ & $38 b-64$ & 195 & $\begin{array}{l}\text { Citation de Mozart } \\
\text { réorchestrée } 10, \\
\text { avec nombreux tropes } \\
\text { ajoutés (voir tableau 4) }\end{array}$ & \\
\hline $338-365$ & & 84 & $\begin{array}{l}\text { Commentaire / dérive } \\
\text { terminale } 10\end{array}$ & $f a-d o-f a$ \\
\hline
\end{tabular}


Au premier coup d'œil, ce tableau fait bien voir l'alternance entre des citations reconnaissables, mais savamment réorchestrées de fragments de l'œuvre de Mozart et des commentaires que j'aime bien appeler des dérives (car on pourrait substituer à la métaphore de la promenade en forêt celle de la navigation). À la première audition, on peut percevoir sans beaucoup de difficultés cette alternance, bien que les frontières entre citations et commentaires puissent apparaître parfois légèrement brouillées, que ce soit parce que les uns et les autres affichent une même subtilité d'écriture instrumentale qui assure une continuité entre les sections, ou à cause de la parenté de matériaux entre une citation et les commentaires qui la jouxtent.

Le tableau 1 révèle également l'analyse relativement classique que Lesage fait du Mozart, et qui se rapproche de la nôtre, résumée dans le tableau 2.

Tableau 2. Analyse formelle de l'Andante cantabile (2e mouvement) de la Sonate K. 330 en Do majeur de Mozart

\begin{tabular}{|c|c|c|c|}
\hline Parties & $\begin{array}{l}\text { Sections (phrases } \\
\text { ou groupes de } \\
\text { segments) }\end{array}$ & $\begin{array}{c}\text { Segments } \\
\text { (Membres de phrases) }\end{array}$ & $\begin{array}{l}\text { Fragments } \\
\text { (incises) }\end{array}$ \\
\hline \multirow[t]{4}{*}{$\begin{array}{l}0-20 a \\
\text { fa majeur }\end{array}$} & $\begin{array}{l}0-8 a \\
\text { Demi-phrase } \\
\text { (antécédent) }\end{array}$ & $\begin{array}{l}0-4 a \\
\Rightarrow \text { demi-cadence }\end{array}$ & $\begin{array}{l}0-2 a \\
2 b-4 a\end{array}$ \\
\hline & & $\begin{array}{l}4 \mathrm{~b}-8 \mathrm{a} \\
\Rightarrow \text { cadence parfaite en } d o\end{array}$ & \\
\hline & $\begin{array}{l}8 \mathrm{~b}-20 a \\
\text { Demi-phrase } \\
\text { (conséquent) }\end{array}$ & $\begin{array}{l}8 \mathrm{~b}-12 \mathrm{a} \\
\text { Séquence } \Rightarrow \text { demi-cadence en } f a\end{array}$ & \\
\hline & & $\begin{array}{l}12 \mathrm{~b}-20 \mathrm{a} \\
\text { Grande chute } \Rightarrow \text { cadence parfaite } \\
\text { en } f a\end{array}$ & $\begin{array}{l}12 b-14 a \\
14 b-16 a \\
16 b-18 a \\
18 b-20 a\end{array}$ \\
\hline \multirow[t]{5}{*}{$\begin{array}{l}20 b-36 a \\
f a \text { mineur }\end{array}$} & $\begin{array}{l}20 b-28 a \\
\text { "Sentence" }\end{array}$ & $\begin{array}{l}20 b-24 a \\
\text { sur pédale en double-croche }\end{array}$ & $\begin{array}{l}20 b-22 a \\
22 b-24 a\end{array}$ \\
\hline & & $\begin{array}{l}24 \mathrm{~b}-208 \mathrm{a} \\
\Rightarrow \text { cadence parfaite en } / a b\end{array}$ & \\
\hline & $28 b-36 a$ & $\begin{array}{l}28 \mathrm{~b}-32 \mathrm{a} \\
\text { Séquence } \Rightarrow \text { demi-cad. en } f a \\
\text { mineur }\end{array}$ & \\
\hline & & $\begin{array}{l}32 \mathrm{~b}-36 \mathrm{a} \\
\mathrm{c} \text { cadence parfaite en fa mineur }\end{array}$ & \\
\hline & & $\begin{array}{l}36 \mathrm{~b}-40 \mathrm{a} \text { Transition de retour } \\
(\Leftrightarrow \text { fa majeur à } 40 \mathrm{~b})\end{array}$ & $\begin{array}{l}36 b-38 a \\
38 b-40 a\end{array}$ \\
\hline \multirow[t]{2}{*}{$\begin{array}{l}40 b-60 a \\
\text { Reprise } \\
\text { intégrale } \\
\text { de o-20a }\end{array}$} & $\begin{array}{l}\text { (mêmes divisions } \\
\text { que plus haut) }\end{array}$ & (mêmes divisions que plus haut) & $\begin{array}{l}\text { (mêmes } \\
\text { divisions que } \\
\text { plus haut) }\end{array}$ \\
\hline & & $\begin{array}{l}60 \mathrm{~b}-64 a \text { Coda } \\
\Rightarrow \text { cadence finale en } f a \text { majeur }\end{array}$ & $\begin{array}{l}60 b-62 a \\
62 b-64 a\end{array}$ \\
\hline
\end{tabular}


En comparant les deux tableaux, on voit tout de suite que les balises citationnelles que Lesage poste tout au long de son œuvre (mes. L13 à 19, 28 à 32, etc.) suivent l'ordre de la partition de Mozart et se confondent, dans le tableau 2, avec le niveau des segments, à l'exception près des deux dernières balises (celles de mes. $\mathrm{L}_{2} 67-270$ et $281-337$ ). L'une ne prend que la première incise de la transition de retour $\left(\mathrm{M}_{3} 6 \mathrm{~b}-38 \mathrm{a}\right)$ et l'autre enchaîne comme un tout la seconde incise de cette transition et l'entièreté de la reprise qui suit. Il s'ensuit un rythme formel où, dans sept cas sur dix, les citations agissent comme des marqueurs d'une durée égale de 24 croches, soit l'équivalent de 4 mesures du Mozart. La courte neuvième citation (12 croches) et la longue dixième ( 195 croches) apportent de la variété dans ce rythme alors que la quatrième (48 croches) témoigne de l'analyse particulière que Lesage fait d'un fragment particulier du «donné Mozart».

Survolons maintenant plus en détails les diverses sections de l'œuvre.

\section{Les neuf premières citations réorchestrées}

Il s'agit bien de citations puisque tout le texte de Mozart (mélodie, rythme, accompagnement, basse) peut s'y entendre ou s'y lire, fut-il éclaté. Il y a très peu d'ajouts ou de modifications à ce texte, et lorsque c'est le cas, ce sera pour faire un lien avec le commentaire précédent: un reste de rythme pointé mes. L140, un accord de piano mes. L158, une sixte ascendante au violon mes. L141, un gruppetto mes. L157. Sur cette donnée de base, Lesage intervient principalement à l'aide d'un certain nombre de procédés d'orchestration.

1) Il y a d'abord la distribution changeante et rapide des notes de la mélodie, de l'accompagnement ou de la basse aux trois instruments du Trio, parfois avec doublure à l'unisson ou à l'octave, parfois avec projection à l'octave et suppression de la note originale. Cette distribution donne naissance, surtout pour les voix intérieures de l'harmonie, à certaines conduites de voix qui s'éloignent des conventions classiques et font donc brièvement ressortir un motif inattendus.

2) À cette distribution s'ajoute la «coloration » de ces lignes principales et secondaires à l'aide de toutes sortes de modes de jeux et articulations: pizzicato, tremolo, harmoniques, ricochet, trilles, glissandi, sul ponticello, sul tasto, arpégiation, etc. Ces modes de jeu exagèrent parfois l'articulation écrite par Mozart (un pizz. remplace un signe de staccato) ou anime considérablement le mouvement, la dynamique et l'agogique en se combinant à une écriture des intensités beaucoup plus détaillée que l'originale.

Il résulte de ces manipulations un effet de fragmentation, de Klangfarbenmelodie assez frappant, qui confère un tout autre caractère, plus instable et disjoint, à l'Andante cantabile galant de Mozart. 
Amplifiant cette altération du caractère, il faut aussi souligner chez Lesage une particularité dans l'écriture des citations, qui sont notées presque toutes selon une métrique de 3/8 plutôt que selon le 3/4 original. Outre qu'elle permet l'intégration de mesures partielles (toutes les citations commencent sur la moitié b des mesures) et qu'elle révèle la perception « hémiolique » du texte original par Lesage (dont la cellule initiale - mes. Mo - est le prétexte), cette écriture maintient également chez les interprètes (et l'auditeur !) une nervosité du tempo, en référant celui-ci à la croche plutôt qu'à la noire.

Voici la $3^{\text {e }}$ citation, qui est un bon exemple de quelques-unes de ces opérations que Lesage effectue sur le texte de Mozart.

Exemple 1. Mes. L65 à 71 (mes. M8b à 12a)



Comme nous le soulignions, la $4^{\mathrm{e}}$ citation est deux fois plus longue ( 48 croches) que la majorité des passages apparentés, et cela s'explique par le fait que Lesage a bien saisi dans son analyse, et voulu laisser intact dans la perception de l'auditeur, le grand mouvement descendant que dessinent, après le saut initial de $7^{\mathrm{e}}$ mineure, certaines notes-clés de la mélodie des mes. Mıb à $20 \mathrm{a}$ (8 mesures). Pour rendre évident cette descente qui s'étend sur un intervalle de $11^{\mathrm{e}}$, on a marqué par un accent, dans l'exemple suivant, ces notes-clés, les autres notes devenant alors leur «ornementation» ou Vordergrund.

Exemple 2. Le mouvement descendant des mes. M12b à 20a

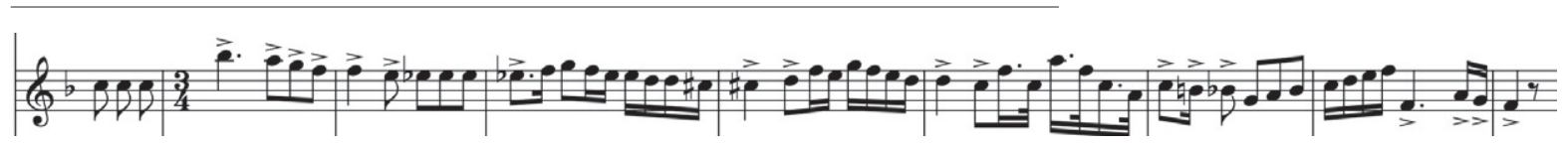


2. On pourrait presque voir des relations proches de celles d'une "série de Fibonacci" $(2,3,5,8,13 \ldots)$ dans cette série de durées en croches: 64 , 24, 96, 36, 126, 60, 142.5, 90, 117, 30, 84, qui, ramenée et arrondie à des multiples de 12, donne la série 5, 2, 8, 3 , (10.5), 5, (12), 7.5, (9.75), 2.5, 7

\section{Les commentaires-dérives}

Ceux-ci sont de longueurs différentes ${ }^{2}$ et mériteront maintenant toute notre attention, puisque c'est le lieu privilégié de l'intervention de Jean Lesage sur le matériau Mozart. La relation avec celui-ci y est parfois assez facile à retracer à l'audition, mais, pour certaines déductions plus recherchées, l'étude de la partition accélérera leur perception.

\section{Introduction}

Un accord de dominante de fa majeur joué pianissimo dès la première mesure dans l'extrême grave du piano, trop grave pour que l'oreille identifie clairement cette fonction, est tenu bien au-delà de la capacité de résonance de ses cordes. Mais celles-ci réverbèrent par sympathie les do de la main droite, d'abord espacés, puis de plus en plus rapprochés, qui déboucheront sur l'énonciation de la cellule initiale en notes répétées du Mozart. Accompagnent cette progression, des bruits blancs, des do aux mêmes octaves ou projetés dans l'aigu, en tremolo ou ricochet, et un motif de 4 te ascendante ( $d o-f a)$ en glissando d'harmoniques qui prépare le premier intervalle significatif du discours qui va bientôt commencer.

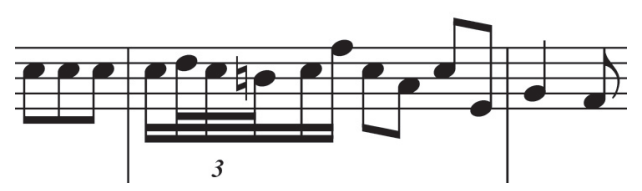

Approche feutrée et graduelle, allusive, du Mozart, mais aussi établissement des «couleurs » particulières que Lesage lui conférera tout au long du Projet.

\section{Commentaire / dérive 1 (mes. 20 à 27)}

Plus court (24 croches), mais beaucoup plus complexe que l'introduction, ce commentaire, désarçonnant, soumet l'auditeur à une épreuve de perception puisque quatre couches instrumentales (le violon, le violoncelle et les deux mains du pianiste) présentent 7 éléments différents, juxtaposés ou superposés. La plupart de ceux-ci sont répétés et présentent, à chacun de leur retour, des différences sensibles. C'est donc dire que la perception est ici fort sollicitée et qu'une première audition ne peut qu'induire un léger vertige... 
Exemple 3. Le commentaire 1 (mes. L20 à 27)
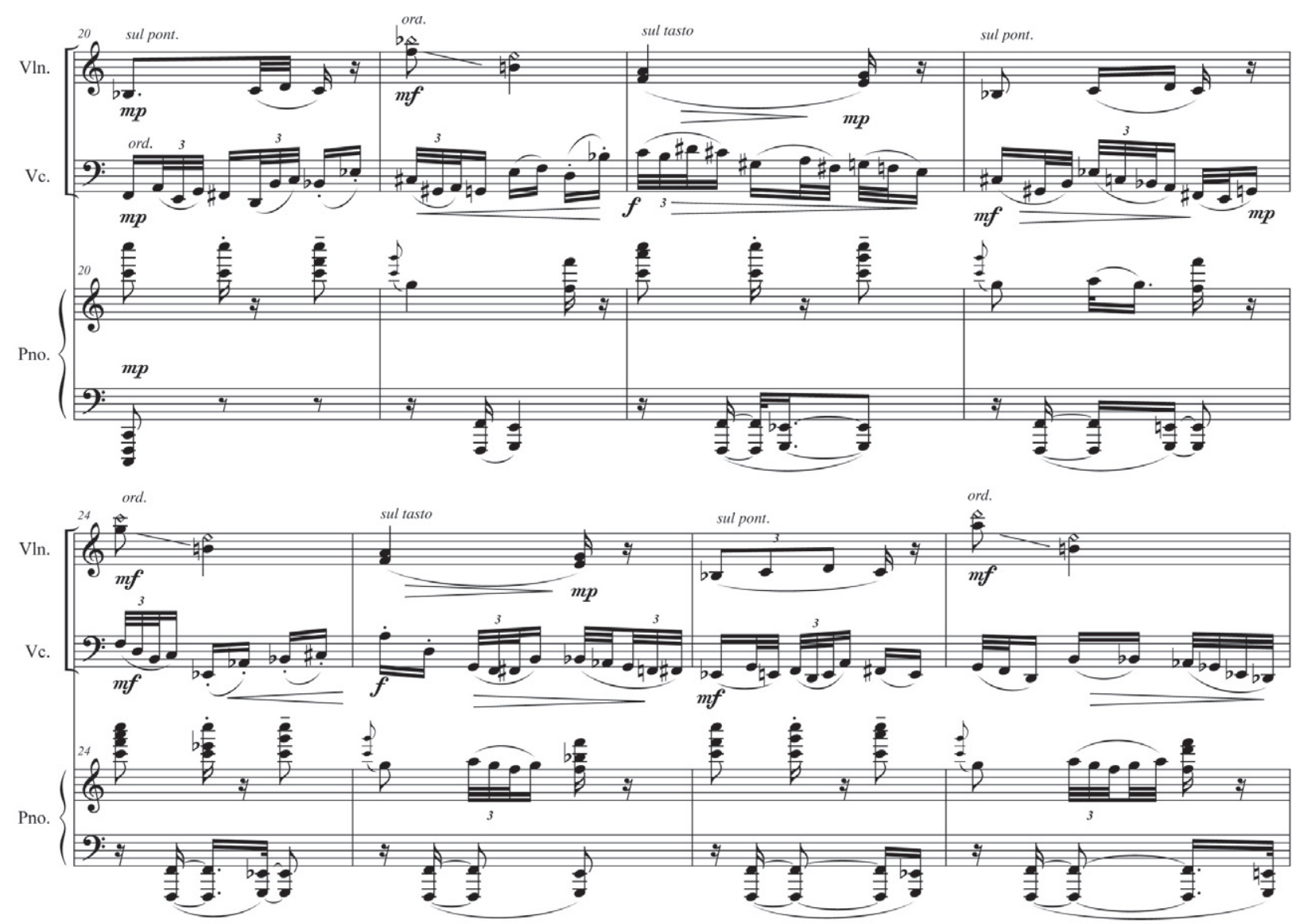

Toutes sortes de techniques sont mises en œuvre dans ces mesures. Considérons le violon : celui-ci alterne entre 3 éléments présentés dans le même ordre: 1) une cellule - broderie issue de la mes. za de Mozart et dont le rythme «s'aplanit» en 3 étapes (mes. Lzo, 23 et 26); 2) un intervalle disjoint descendant en harmoniques, que l'on pourrait dire issu de la 6te descendante de la mes. M1, car ses trois versions (mes. L 21, 24 et 27), croissantes quant à leur déplacement (triton, 6te mineure, $7^{\mathrm{e}}$ mineure), se terminent toutes sur une note à fonction de sensible, ici sur un si bécarre, sensible du do dont nous entendrons l'accord à la mes. L28, où débute la $2^{\mathrm{e}}$ citation; 3 ) la succession appogiature - résolution en zces parallèles, immuable, directement prise de la mes. M4a. La main droite du piano alterne pour sa part entre 
2 éléments: 1) la cellule initiale des do répétées (mes. Mo), se densifiant peu à peu en accord ; 2) une autre formule appogiature - résolution (mes. Mza) avec un rythme de longue / brève qui est progressivement monnayé par l'interpolation du gruppetto de Mozart (mes. Mia). La main gauche du piano répète son seul élément (que l'on peut relier à divers mouvements conjoints de deux notes dans les premières mesures du Mozart), rythmé selon un petit jeu mathématique de durées (en triples-croches: $2+8,3+7,4+6,5+5,6+4$, $8+2,9+1)$. À ces 3 couches, qui ont chacune leur propre rythme d'alternance (par 3 mesures, 2 ou 1), s'ajoute celle du violoncelle, qui déroule une ligne dodécaphonique animée sur les 8 mesures. Son intensité lui donne une fonction de premier plan, et son organisation rythmique interne le détache du carcan de la mesure de 3/8 (si l'on compte les temps d'un gruppetto de triolets de triples à l'autre, on trouve la série de durée suivante, en croches: $1,2,3,4,2,4,1.5,1.5,5)$.

Il fallait regarder en détail ce premier commentaire pour prendre déjà conscience de l'hétérogénéité non seulement des techniques de Jean Lesage mais surtout des éléments de son langage. Ici des traits caractéristiques de la musique tonale (la majorité des éléments s'entendent en fa ou à sa dominante) sont carrément superposés à de la musique atonale, pour produire un brouillage efficace de notre perception entre les deux premières citations de Mozart, tout en entretenant des liens avec celles-ci. Dérive contrôlée avec visibilité moyenne...

Commentaire / dérive 2 (mes. L33 à 64)

Ce commentaire, au contraire du précédent, privilégie la clarté harmonique. Toutes les notes entendues font partie d'une échelle adaptant dans le tempérament égal la série des 20 premiers harmoniques naturels d'une fondamentale de $f a$ ( $f a 1$ au violon et aux deux mains du piano, $f a \circ$ au violoncelle) puis de do (do 1, sauf au violoncelle: do o). La «modulation» de $f a$ à do a lieu à la mesure L50, sauf pour la main gauche du piano (mes. L54). Le passage devient en quelque sorte hyper-tonal puisque hyper-polarisé, et il reproduit ainsi de façon magnifiée la progression tonique vers la dominante que les 8 premières mesures de Mozart ont affichée.

Par contre, sur le plan mélodique, les rapports thématiques sont plus diffus. Le violon et le violoncelle décrivent des courbes mélodiques sinueuses où s'entendent quand même quelques motifs de gruppetto et d'arpèges entendus aux mesures Mi et 5. Mais nous ne suivons plus un développement systématique de ceux-ci comme lors du premier commentaire. Le passage semble organisé plus librement. Cependant, on peut remarquer que ces 


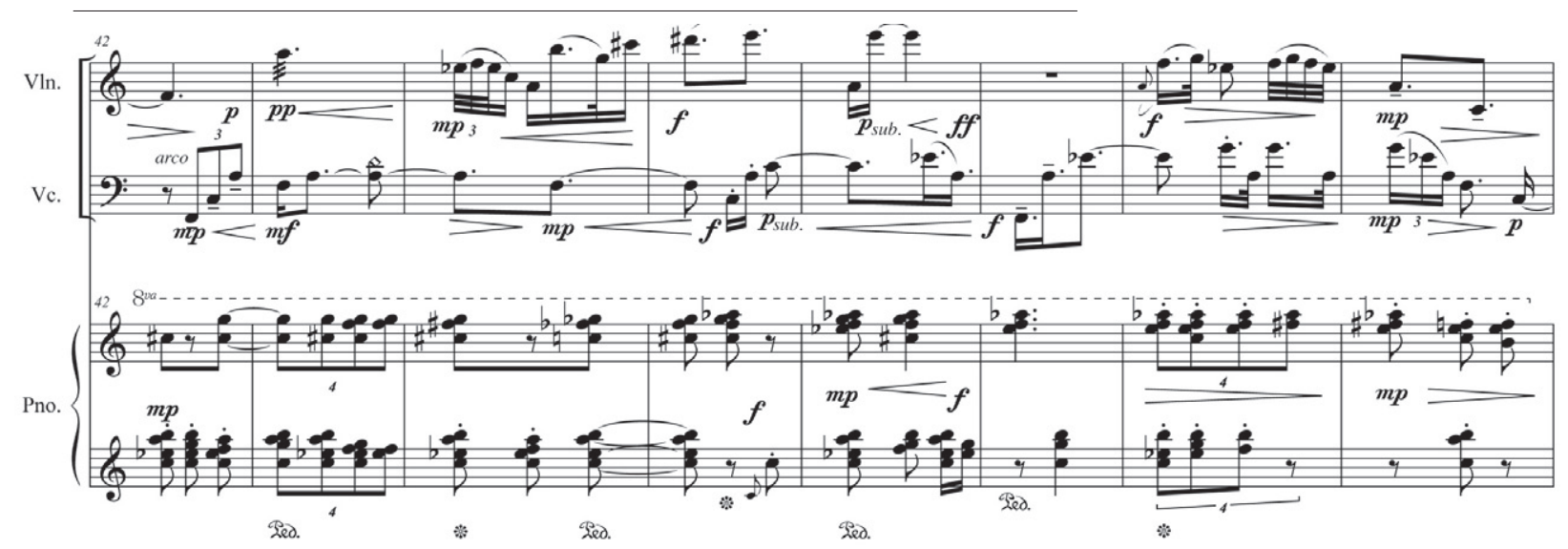

courbes ont un profil globalement ascendant puis descendant (à rapprocher de celui des mesures M6, 7 et 8) généralement accompagné d'un crescendo puis d'un diminuendo. Cela nous a incité à constituer ces profils en unités phraséologiques distinctes dont la longueur variable semble s'approcher des proportions de la série de Fibonacci classique (1, 2, 3, 5, 8, 13, 21, 34, etc.). Ainsi, au violon, à partir de mes. 35 : courbes de 3,5 et 8 mesures sur $f a$, puis de 14 mesures sur do, cette dernière sans diminuendo; au violoncelle : à partir de mes. 42 : courbes de 3 puis 5 mesures sur $f a$, et plus loin, de 8 mesures sur do. Le piano semble lui aussi organiser ses unités de même manière (à partir de mes. 36 : unités de $8,5,4,12,22,3$ et 33 croches) en signalant à chaque fois leur début par une petite note rapide et forte, ce qui renvoie à un ornement similaire de la mesure $\mathrm{M}_{5}$. Spéculation de notre part? Après consultation de l'auteur, il semble que toutes ces belles proportions aient été introduites de façon purement intuitive...

\section{Commentaire / dérive 3 (mes. $\mathrm{L}_{72}$ à 83 )}

Ce commentaire adopte une forme en arche sur les plans du rythme et des intensités: accelerando doublé d'un crescendo, suivi d'un ralentando diminuendo. Pour Lesage, l'idée d'accéléré - ralenti est contenue dans le

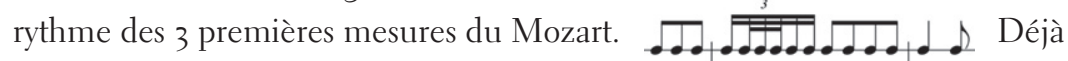
effleurée dans les légères fluctuations de vitesses entendues au piano dans le commentaire précédent (intervention épisodiques de quartolets de croches), cette idée se matérialise ici pleinement à ce même instrument sous la forme d'une progression du nombre de ses attaques par mesure de $3 / 8$, selon une courbe croissante puis décroissante: $3,4,5,6,7,8,9$ puis $8,7,6,5,4,3$. 
3. Les intervalles sont ici notés en nombre de demi-tons: 1 = seconde mineure; 2 = seconde majeure; 3 = tierce mineure; 4 = tierce majeure; etc.
Autre exemple de déduction révélatrice de l'analyse d'un petit fragment du Mozart par Lesage :



le déplacement en mouvement contraire (symétrique) des deux mains dans le motif d'accords homorythmiques de ces mes. M9 à 11 et la structure symétrique de l'un de ces accords, en $5^{\text {te }}$ diminuée, entraîne l'idée de construire symétriquement les accords entendus au piano pendant cet accéléré - ralenti, c'est-à-dire que leur structure intervalique donne la même suite d'intervalles, que l'on lise celle-ci de bas en haut ou de haut en bas. Ces accords peuvent structurellement se ramener à 4 : dans l'exemple ci-dessous, les accords A présentent une structure $3223^{3}$, les B en sont une variante (32423), et les C encore une autre (33233) et ces trois familles d'accords peuvent toutes trouver leur origine dans l'accord de 5te diminuée do-mib-fa\# (33) de la mes. Mgb ou dans le renversement de l'accord de dominante de Miob (32). L'accord D a pour sa part comme origine le premier accord de la mes. Mio (524 de bas en haut).

Exemple 5. Mesures L72 à 76

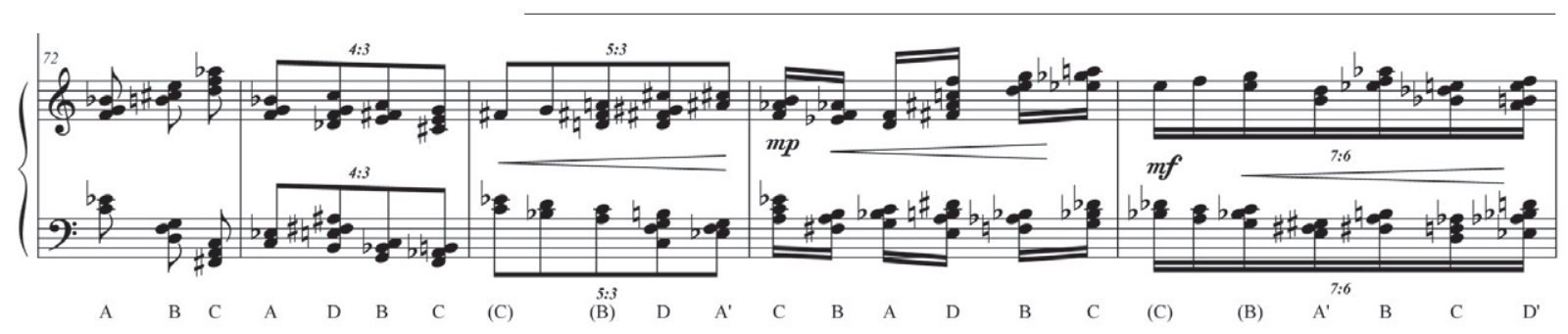

Remarquons au passage deux petites altérations de cette logique: les accords A' et D', dont la symétrie est déformée, et l'insertion de brèves citations textuelles de Mozart, soit les 3 premiers accords des mes. L74 ou 76 qui proviennent des mes. Mya et $10 b . .$.

Pendant ce temps, violon et violoncelle extraient certaines des notes de la partie de piano et les jouent en homorythmie avec celui-ci jusqu'au climax rythmique, puis s'en détachent. À partir de ce point, toute cette succession est jouée en rétrograde (!), sans autre modification que la transposition à la 5te affectant la première mesure (L79) de cette rétrogradation. 
Cette dérive, la seconde plus longue, se divise en trois séquences (comme les appelle Lesage). La première développe le mouvement descendant mentionné à l'exemple 2 et que la $4^{\mathrm{e}}$ citation vient de nous faire entendre dans son intégralité. Quatre chutes successives sont entendues, chacune étant constituée par des traits descendants joués simultanément par le violon et le violoncelle en hétérorythmie. Ces chutes expriment des portions de série harmonique, les deux premières chutes (figurant à l'exemple suivant) étant basées sur des fondamentales de do, les deux autres sur sol. Le piano ponctue ces chutes en exposant, souvent dans un mouvement contraire des deux mains du centre vers les registres extrêmes, des accords qui regroupent des sons conjoints de ces mêmes séries.

Exemple 6. Mesures L98 à 105, parties de violon et violoncelle

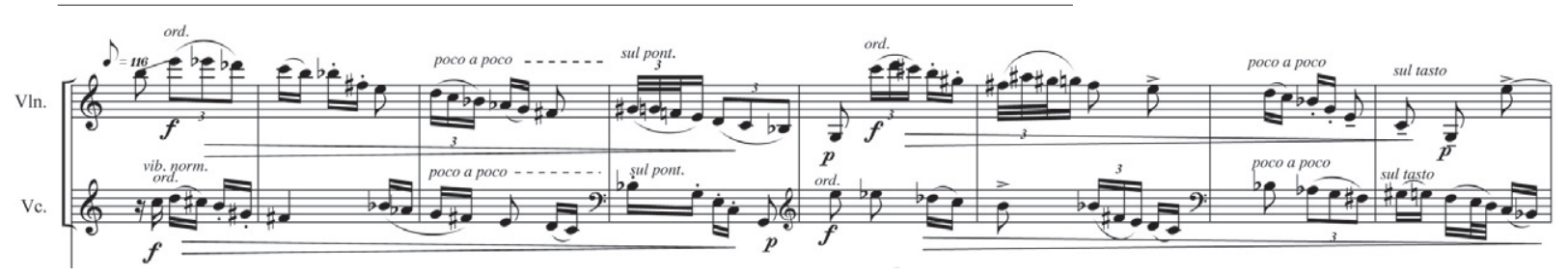

Après une courte stabilisation (mes. L116-118) dont la dernière mesure nous fait moduler vers les harmoniques d'une fondamentale de sib, la seconde séquence amorce un mouvement ascendant qui se poursuivra dans la séquence suivante. On pourrait retracer l'origine de cette ascension dans le texte mozartien lui-même, à la fois dans la gamme ascendante de la main gauche de la mes. M13 ou dans celle de la main droite des mes. M18b et 19a précédent la cadence (cf. exemple 2). Cette deuxième étape est caractérisée par les enflés successifs en tremolos de cordes, déduits sans doute du trille entendu mes. M15a. Le piano se joint ici au mouvement ascendant. L'étape suivante, la troisième séquence, continue l'ascension en développant cette fois-ci la formule rythmique pointée de la mes. Mı8b, qui est juxtaposée et

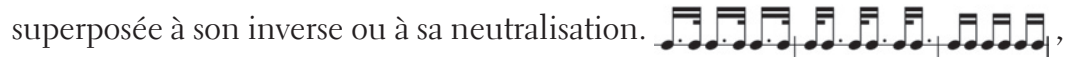
alors que l'harmonie revient à une polarisation orientée vers une fondamentale de fa, exprimée explicitement au début de la citation qui suit.

Les 5 commentaires qui suivent s'inscrivent entre les citations de segments de la partie mineure de l'Andante de Mozart et de la transition qui ramène la reprise de la $1^{\text {re }}$ partie. 
Sur la pédale de fa répétée en doubles-croches (avec des restes de rythmes pointés hérités du commentaire précédent, ce commentaire fait entendre des motifs déduits des citations de divers segments de la partie mineure: sixtes parallèles provenant de M21b (à L148, 149 et 157, gruppetto de M26a (à L150, 151, 152 et 156-157), itérations avec appogiatures de M25b (à L152), ligne de basse ascendante de $\mathrm{M}_{31}$ (à L151), auxquels se superposent quelques groupes d'accords symétriques rappelant le commentaire 3. Certains de ces motifs déstabilisent légèrement le tempo par l'irrationalité de leur rythme. Une courte sinuosité en tremolo sul ponticello quasi dodécaphonique au violon (L153, que le violoncelle imite immédiatement par renversement !) peut, par le brouillage harmonique qu'il induit, rappeler le passage du violoncelle de L2O-27 de fonction similaire. Ce brouillage était déjà en germe dans l'hésitation majeur-mineur de L147-148, dans les accords symétriques du piano à L150 et dans le petit cluster chromatique (Do\# à Fa) qui résulte de la superposition des différents motifs à L 152. Le retour de la pédale de Fa (à L155) va assurer une continuité sans couture avec la citation suivante, annoncée par l'imitation de la cellule ascendante de L24b assortie d'un gruppetto.

Exemple 7. Mesures L152-155

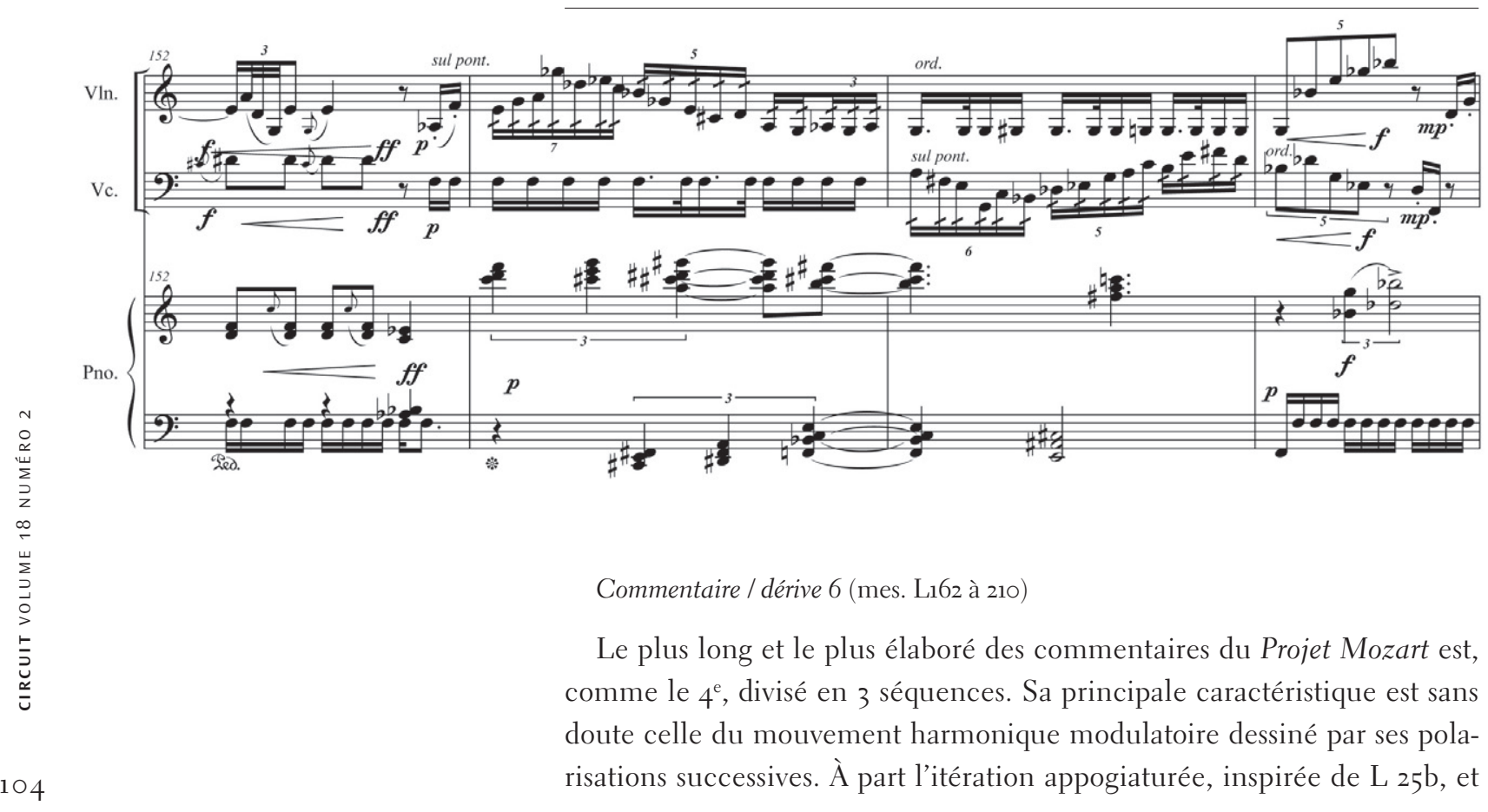


qui agit ici comme charnière formelle (à L184, 180 et 184-189), les motifs mélodico-rythmiques qui articulent ce mouvement paraissent secondaires, simple expression de séries harmoniques rapportables à des fondamentales qui reproduisent les fonctions entendues dans la deuxième partie de l'Andante, en fa mineur et son relatif, lab majeur. Ainsi, la première séquence oscille entre réb et sa broderie do, comme dans les mes. M31-32 ou 33-34. Pour la seconde, après des fondamentales claires fa et réb (cf. M3ob-31a), la musique fige sur une couleur plus ambiguë de notes contradictoires dont la note grave, mib, n'est pas une fondamentale convaincante. Enfin, une musique plus feroce, fortissimo, malmène la perception métrique, à l'instar de ce passage en canon rythmique des mes. M32b-34a. En plus d'accents déstabilisants, on oscille entre le $3 / 8$ et ses compressions ( $5 / 16$ et 2/8). Pendant ce temps, nous parcourons sur le plan harmonique un cycle qui part de mib et traverse $f a, s o l, d o, l a b$, sib et réb avant de revenir au mib (diminuendo), «dominante» du lab qui débutera la citation suivante. Le violon et la main droite du piano expriment des harmoniques plus élevés (jusqu'au $20^{\mathrm{e}}$ ) alors que le violoncelle et la main gauche du piano se concentrent sur des harmoniques de moindre rang. Les durées des fondamentales successives, calculées en doubles-croches (mais avec des débuts arrondis à la mesure près, étant donné les décalages ou les brèves hésitations de l'un ou l'autre instrument lors de son entrée dans l'harmonie suivante, - ce qui donne d'ailleurs d'intéressants flous), donnent une série démontrant, malgré ses petites irrégularités, un mouvement global de compression suivie d'une dilatation: 32, 13, 15, $4,5,11,5,16$. Les pôles de plus longue durée sont mib, fa, sol, sib et mib... Nous parlions d'hypertonalité...

Exemple 8. Mesures L201-205

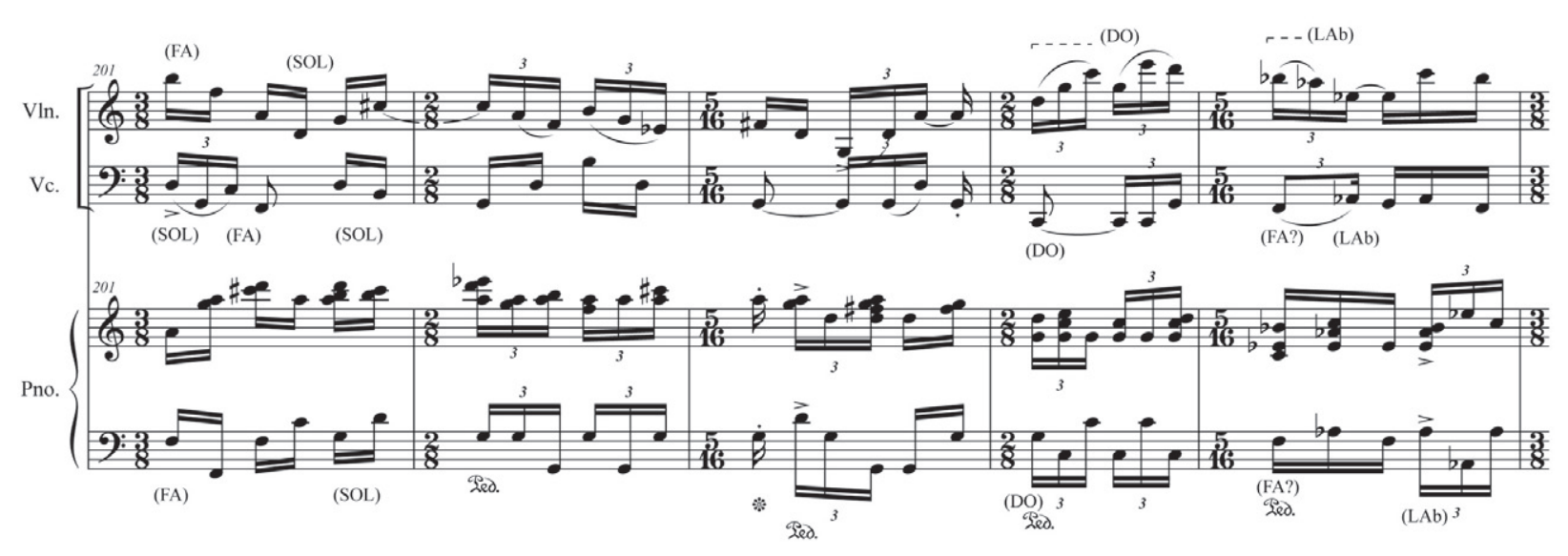


Si l'on excepte un passage en accords très marqués ( $\left.\mathrm{M}_{34} \mathrm{~b}-36\right)$, les citations 7 et 8 se réduisent presqu'à une diaphonie et donnent sa place au silence lors du court canon rythmique évoqué plus haut (M32b-34a). Le commentaire qu'elles encadrent magnifiera cette raréfaction en accentuant la fragmentation du discours. Celle-ci est remarquablement bien contrôlée pour que nous percevions le rapport maintenu, dans le vide, avec le texte original de Mozart. Harmoniquement et mélodiquement, nous avançons et reculons dans notre souvenir du texte original, selon ce qu'une simple note isolée, une basse ou un accord de 2 ou 3 sons nous suggèrent, Lesage y jouant même très habilement avec le double sens, comme nous le révèle la comparaison entre le segment de Mozart (où toutes les notes ont été chiffrées selon leur ordre d'arrivée, les notes simultanées portant le même numéro) et le commentaire 7 .

Exemple 9. Mesures M28b à 36a

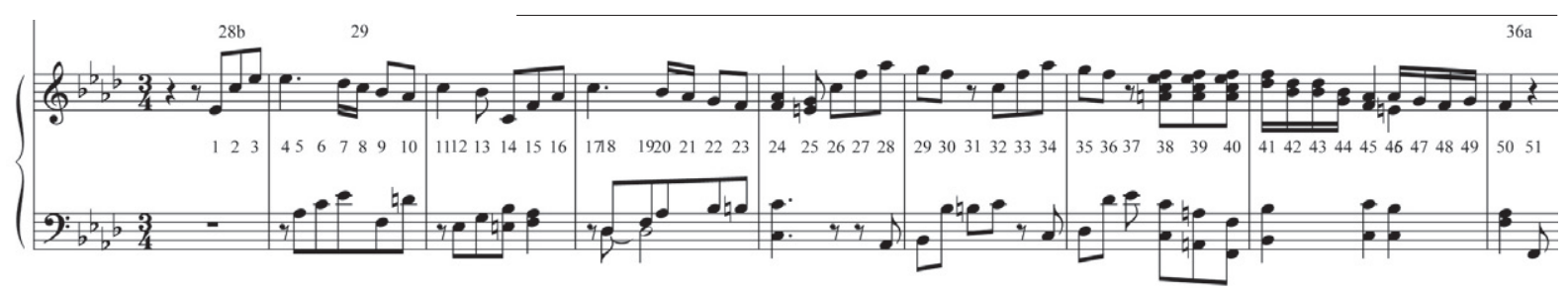

Exemple 10. Mesures L215 à 230






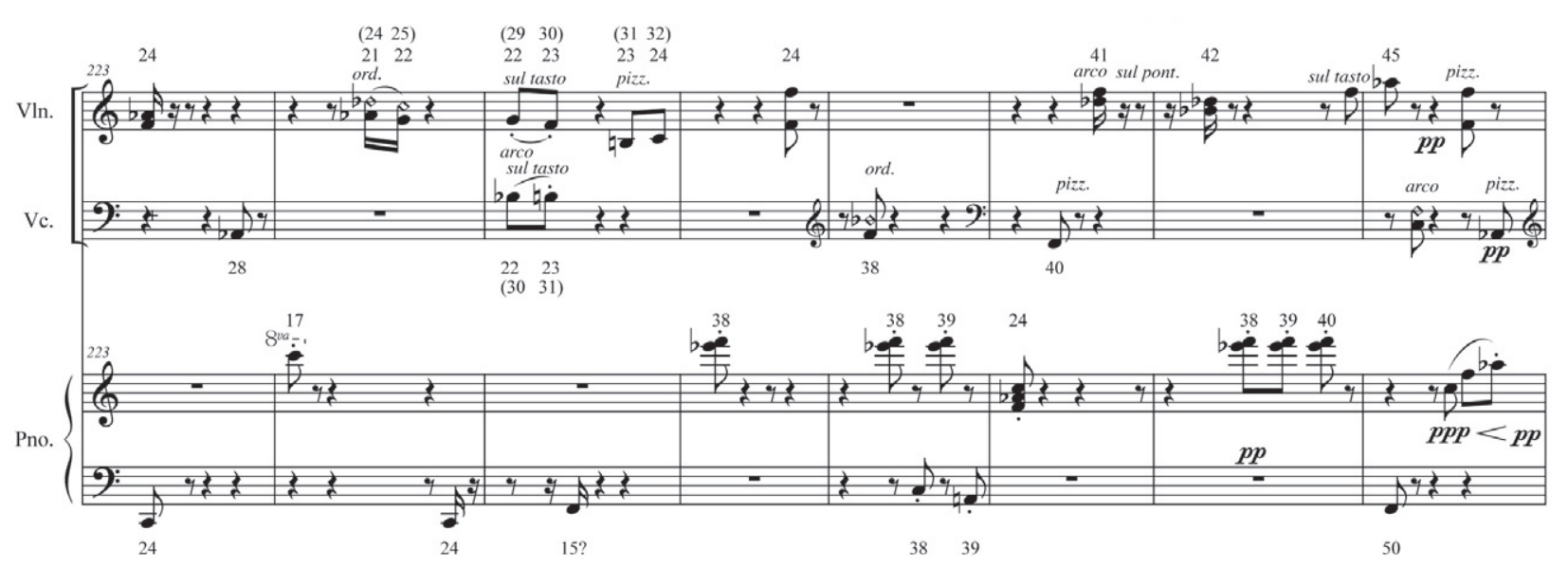

Commentaire / dérive 8 (mes. L235 à 266)

Ici sont développés en 5 courtes séquences quelques éléments que l'on vient d'entendre dans la citation précédente. Ce sont d'abord des arpèges parfois brisés déduits de L28b, 3ob, 32b et 33b (main droite), de même que de L29 (main gauche). Ils sont articulés en syncopes de doubles-croches (dans les séquences 1, 2 et 5 piano contre cordes, puis dans les séquences 3 et 4 , au piano, main droite contre main gauche), syncopes sans doute inspirées par le jeu d'imitation du canon rythmique de $\mathrm{M}_{32}$ b-34a. Le mouvement de ces arpèges est globalement ascendant, sauf pour les séquences 3 et 4 où les arpèges brisés prennent plutôt l'allure de petites cellules de 2 ou 3 notes disjointes, exposées au violon (L251-261) ou en dialogue entre les deux mains du piano. Ces arpèges ou cellules peuvent être interrompues: 1) soit par des descentes en doubles-croches ou en doubles-croches de triolets (que l'on peut voir comme une extension des petites descentes de 2 doubles-croches de M29 et 31 qui suivent l'arpège ascendant dans le texte de Mozart); 2) soit par des accords staccato au piano (renvoyant aux trois accords forte de $\mathrm{M}_{34} \mathrm{~b}$ ) ; 3 ) soit par une combinaison des deux précédents. Tous ces éléments expriment de nouveau des segments de séries harmoniques, principalement sous la forme d'un mode octaviant regroupant les harmoniques 8 à 15. Le tableau suivant, à lire de gauche à droite et de bas en haut, donne la suite de ces éléments avec la fondamentale qu'ils expriment et, entre parenthèses, la durée qu'ils occupent, calculée en croches. 
Tableau 3. Succession des éléments du commentaire / dérive 8

\begin{tabular}{|l|l|l|}
\hline Arpèges & Descentes & Accords \\
\hline lab (10) & lab, en doubles (3) & lab (3) \\
\hline sib (15) & sib, en doubles de triolets (4) & sib (4) \\
\hline$r e ́ b(21)$ & ---- & $l a b(3)$ \\
\hline$d o(30)$ & $f a(3$ en doubles +5 en doubles de triolets) & ---- \\
\hline$f a(10)$ & ---- & ---- \\
\hline
\end{tabular}

L'arpège de fa terminal mène tout naturellement à l'accord de fa mineur qui commence la citation suivante.

Commentaire / dérive 9 (mes. L271 à 280)

Ce dernier commentaire coupe en deux la transition dont Mozart se sert pour revenir en $f a$ majeur et amener à la reprise de la première partie de l'Andante. Il consiste essentiellement en un jeu de permutations des sixtes parallèles entendues en $\mathrm{M}_{3} 6 \mathrm{~b}-38 \mathrm{a}$, accompagné par une répétition à la basse de doubles-croches tournant autour de la pédale de fa caractéristique de cette transition. Cette broderie du $f a$, entendue en $\mathrm{M} 23$ et donc dans L145-146, n'avait pas été développée pendant le commentaire 5. C'est ici que Lesage s'en occupe, alors que le texte de Mozart ne l'aborde que dans une voix intérieure (le mi de $\mathrm{M}_{39}$ ) et non à la basse.

\section{La dernière citation, avec tropes (mes. L281 à 337)}

Cette dernière unité formelle (281-337) interrompt la succession citation réorchestrée / commentaire - dérive qui a marqué jusqu'ici le déroulement formel. Pour cette reprise de la première partie de l'Andante, Lesage n'interrompra plus le texte de Mozart que par de brefs tropes (ajouts interpolés) de une ou de deux mesures, insérés entre les segments ou incises du Mozart réorchestré. Ces tropes prolongent un accord pour l'amplifier en s'inspirant de la série harmonique déduite de la basse de cet accord, ou développent brièvement un comportement motivique que l'on vient d'entendre. Mais, reprenant à son compte la tradition baroque et classique de varier les reprises, Lesage pousse plus loin son intervention sur le texte de Mozart lui-même en ajoutant aux procédés de réorchestration déjà évoqués plus haut des variations mélodiques (ajouts d'échappées, de notes de passage, de gruppettos supplémentaires) et rythmiques (entre autres par l'utilisation fréquente du rythme pointé trochaïque de $\mathrm{M}_{17-18}$ et de sa forme inversée, iambique). S'ajouteront à ces variations des « contaminations » par des références à des «matériaux Lesage » déjà entendus dans les commentaires / dérives précédents: amplification des 
accords (surtout, de nouveau, par imitation du modèle de la série harmonique) ou syncopes inspirées du commentaires 8 (L293).

Il résulte de tout cela une fantaisie, voire un humour et une légèreté que Mozart lui-même (si l'on se fie à ce que l'on connaît de sa personnalité) n'aurait pas dédaignés... Nos attentes vis-à-vis du texte mozartien vont être joyeusement perturbées, notamment sur le plan de la perception de la métrique, constamment altérée par les tropes.

Tableau 4. Plan de la dernière citation, avec description des tropes

\begin{tabular}{|c|c|c|c|}
\hline Mesures & $\begin{array}{l}\text { Mesures } \\
\text { du Mozart }\end{array}$ & $\begin{array}{l}\text { Durée en } \\
\text { croches }\end{array}$ & Description des tropes \\
\hline $281-284$ & $38 b-40 a$ & 12 & $-\cdots$ \\
\hline $285-286$ & & 6 & $\begin{array}{l}\text { Annonce de la cellule initiale (do répété) } \\
\text { articulée en jeu de hoquet. }\end{array}$ \\
\hline $287-289$ & $40 b-42 a$ & 12 & ----- \\
\hline $290-291$ & & 6 & $\begin{array}{l}\text { Gruppettos en imitation; appogiature - } \\
\text { résolution en séquence ascendante; pour } \\
\text { suite du jeu de hoquet. }\end{array}$ \\
\hline $292-295$ & $42 b-44 a$ & 12 & ----- \\
\hline 296 & & 3 & $\begin{array}{l}\text { Légère extension de la durée de l'accord de } \\
\text { dominante }(d o) \text {, magnifié au piano en accord } \\
\text { de série harmonique. }\end{array}$ \\
\hline $297-301$ & $44 b-48 a$ & 24 & $-\cdots---$ \\
\hline $302-303$ & & 6 & $\begin{array}{l}\text { Autre extension de l'accord de } d o \text { harmonique } \\
\text { au piano, avec traits ascendants aux cordes, } \\
\text { déduits de M47a. }\end{array}$ \\
\hline $304-310$ & $48 b-52 a$ & 24 & ----- \\
\hline $311-312$ & & 6 & $\begin{array}{l}\text { Amplification du mouvement appogiature } \\
\text { - résolution descendante }\left(M_{52 a} \text { ) par la }\right. \\
\text { continuation de la descente et un léger } \\
\text { ralentissement du rythme (piano main } \\
\text { droite): } L 310=4+2 \text { doubles-croches; } L_{311-312} \\
=5+3 \text { doubles-croches. }\end{array}$ \\
\hline 313 & $53 a$ & 3 & $\begin{array}{l}\text { (Compression de la montée de main gauche } \\
\text { de M53a sur } 3 \text { croches) }\end{array}$ \\
\hline $314-316$ & (53b) & 6 & $\begin{array}{l}\text { Extension de la descente de main droite de } \\
\text { M53b sur } 3 \text { mesures. }\end{array}$ \\
\hline 317 & $54 a$ & 6 & $-\cdots--$ \\
\hline 318 & & 3 & $\begin{array}{l}\text { Accord harmonique sur sib qui, en se basant } \\
\text { sur la basse de l'accord de M54a, détourne } \\
\text { brièvement la fonction de celui-ci. }\end{array}$ \\
\hline $319-321$ & $54 b-56 a$ & 12 & ----- \\
\hline 322 & & 3 & $\begin{array}{l}\text { Ajout d'une transition mélodique au violon } \\
\text { développant les appogiatures sur rythme } \\
\text { iambique; extension de l'accord de sib } \\
\text { harmonique au piano avec rythme irrationnel } \\
\text { (cf. les quartolets du commentaire } 2 \text { ). }\end{array}$ \\
\hline $323-319$ & $56 b-60 a$ & 24 & -..-- \\
\hline 330 & & 3 & $\begin{array}{l}\text { Petite prolongation de l'accord de } f a \\
\text { harmonique. }\end{array}$ \\
\hline $331-327$ & $6 o b-64 a$ & 24 & ----- \\
\hline
\end{tabular}


L'exemple suivant (mes. M304-317) témoigne de quelques-unes de ces variations et montre l'un des tropes (L311-312). On y observera aussi la seule intervention qui altère le texte de Mozart par suppression : après la compression sur 3 croches, à $\mathrm{L}_{313}$, de la montée de la main gauche de $\mathrm{M}_{53} \mathrm{a}$, la descente la-sol-fa de la M53b à la main droite est ici remplacée par une extension tropée de cette descente sur 3 mesures (L314-316, donc 9 croches). La descente la-sol-fa reste cependant encore visible et audible au violon (dernières croches de chaque mesure).

Exemple 11. Mesures L304 à 317



2ed.
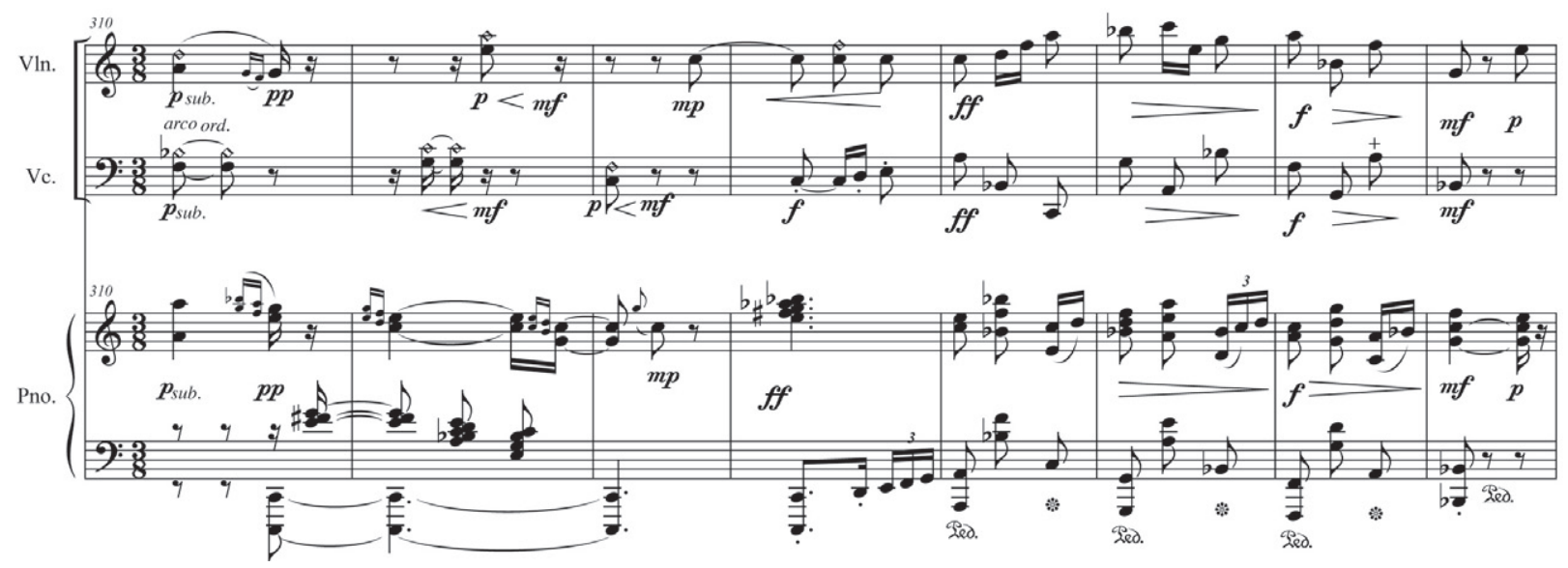

Commentaire / dérive 10 (mes. L338 à 365$)$

Dernier commentaire qui peut très justement être considéré comme cadentiel en ce qu'il imite, en se référant à trois fondamentales, une progres- 
sion typique d'une cadence parfaite : du fa de L343, on passe à do (perceptible surtout au piano à L 342 , alors que le violoncelle s'aventure plutôt vers sol; puis on retourne clairement à fa à partir de L350 (mais un fa où l'harmonique 11 n'est plus interprété toujours comme un $s i$, mais parfois comme un sib). Des gruppettos et des figures arpégées articulent un mouvement d'abord globalement ascendant au violon et au violoncelle, et le tout se termine par une série de clusters d'harmoniques aigus répétés au piano, croches contre doubles-croches, alors que la tierce fa-la tenue dans l'aigu des cordes, monte encore et se perd.

\section{Le projet Mozart et le projet Lesage}

Dans une conférence (inédite) qu'il a prononcée à Saint-Irénée en 2000, Jean Lesage a cherché à présenter les sources esthétiques de son travail, à mettre au clair le pourquoi de celui-ci. Articulée autour des thèmes 1) de l'actualité du Baroque, 2) de l'intertextualité en rapport avec la problématique de la citation, 3) du temps et de la mémoire et 4) des représentations post-modernes, cette conférence recèle quelques phrases qui nous permettent de situer le projet Mozart au sein de que l'on pourrait bien appeler «le projet Lesage».

Ainsi, dans le cours d'une discussion sur l'opposition entre le style de la Renaissance et celui du Baroque, Lesage mentionne les caractéristiques de ce dernier qui l'ont séduit:

Le Baroque [...]offrira une fuite hors du réel vers le merveilleux, le prodigieux, le fantastique, le surnaturel.

L'équilibre [y] est perturbé par les mouvements excessifs. La réserve distinguée fait place à l'héroïsme violent et à la sensualité. Le contrôle de soi est remplacé par une formidable conscience de soi.

Puis, il énumère certains traits du discours baroque qu'il a cherché à transposer dans son propre langage :

successions rapides d'idées musicales antagonistes, conception formelle en mosaïque, brusques variations de climats et de mouvements, discours en perpétuelles ruptures, traitement harmonique et rythmique diversifié, qui dévoile un goût marqué pour l'artifice et l'emphase théâtrale.

De façon plus globale, pour Lesage, intégrer cette influence, c'est travailler à une «esthétique de la discontinuité, [à un] art de l'instabilité généralisée». «Le discours [baroque] est non directionnel; c'est une musique de l'instant présent, qui n'a pas de début officiel, pas de point d'aboutissement nécessaire.» «Seul le plaisir de l'errance est conservé.» Il s'agirait finalement d'une «dynamique temporelle non téléologique.» 
Nul doute que ces caractéristiques s'appliquent à de nombreuses œuvres de Lesage. Il y a un peu de tout cela également dans Le projet Mozart, comme on l'a souligné dans cette analyse: exagération de l'articulation mozartienne, hypertrophie des fonctions harmoniques, brouillages, etc. Mais, si cette œuvre s'offre à l'auditeur comme une remarquable démonstration d'invention, de fantaisie, de contrastes, baroque même si son substrat est classique, cette démonstration est d'autant plus intéressante que le référent qu'elle fait déraper, à partir duquel elle dérive, est ici clairement exposé, entendu.

Des œuvres comme Les sensations confuses (titre approprié, s'il en est !) peuvent dérouter, car les référents sont réduits à l'état de fragments, de bribes, d'accords isolés, d'allusions fugitives, gestes qui sont juxtaposés rapidement selon une logique formelle qui serait, selon les mots de Lesage, une «transposition de la trame narrative du rêve dans le champ du sonore». L'errance et l'absence de téléologie est, depuis l'effondrement des certitudes, une thématique privilégiée de l'art: irrésolution, dislocation, disjonction, distance face au matériau, etc. Que cette errance ait toujours été source de plaisir n'est pas sûr, et l'art moderne s'est plus d'une fois fait accuser de ne parler que d'angoisse (bien qu'il n'ait pas à revendiquer un tel monopole, comme de nombreux exemples de l'histoire peuvent le prouver). Paradoxalement, une œuvre comme Le projet Mozart m'apparaît à certains égards comme une exception dans cette attitude.

Dans la musique à programme, on le sait, le référent est source de plaisir en ce qu'on l'identifie comme la source de l'inspiration, bien sûr, mais aussi en ce que, s'y appuyant, on peut «l'oublier» pour alors suivre le travail spécifiquement musical dont il est le prétexte. Dans Le projet Mozart, il y a un a priori formel de citer le texte de Mozart intégralement et dans l'ordre original, par segments successifs. Les citations de Mozart deviennent alors comme des îlots bienvenus, fussent-ils allègrement et irrévérencieusement triturés, points de repères que l'on quitte aussitôt pour une autre dérive, bien contrôlée malgré ses excès. Le plaisir naît de la reconnaissance du travail de réorchestration dans les citations et aussi de celui effectué dans les commentaires qui suivent celles-ci: déductions motiviques et agogiques, écho volontaire des fonctions tonales, etc. S'il y a errance ici, c'est une dérive ludique avec carte et boussole.

En plus d'imposer un certain rythme (rassurant?), les citations charrient aussi avec elles une téléologie, un but, qui est celui de toute musique tonale, soit la cadence finale, terme d'un voyage dont les étapes auront été les cadences intermédiaires. L'errance à travers progressions d'accords, emprunts et modulations est toujours résolue par un retour au port d'attache. Des attentes 
sont donc là aussi créées, particulièrement chez l'auditeur informé, jusqu'à l'ultime accostage. Dans Le projet Mozart, la téléologie tonale n'est pas abolie puisque le trajet mozartien est entièrement entendu, mais cette téléologie est attentivement reconsidérée. Les passages basés sur des fondamentales et leur série harmonique deviennent le lieu d'inventions figuratives autour d'un seul accord équivalant à une seule «hypertonalité»: statisme agité. Le commentaire 7 fragmente habilement la perception tonale: avancée et recul dans les fonctions attendues. En ce qui concerne la cadence ultime - pour ne parler que de celle-ci - Mozart n’a pas ici le dernier mot: celui-ci revient à Lesage, qui décide non de contredire, mais plutôt d'amplifier celle de son célèbre prédécesseur pour l'amener à se résorber enfin dans une zone où toute fondamentale devient possible: un cluster d'harmoniques dans l'extrême aigu... L'errance n'est pas ici instabilité comme posture esthétique, ou évocation d'angoisse, mais devient véritable plaisir, ouverture aux surprises du voyage.

Pour reprendre la métaphore du rêve, il s'agirait plutôt de ce genre de rêve à la frange du réveil, où le rêveur peut encore, dirait-on, intervenir (à son bénéfice...) dans le cours des choses.

Dans la conférence précitée, Lesage a bien résumé son entreprise de recontextualisation du passé.

Le travail de la mémoire est au cœur de mes préoccupations artistiques. Dans mes travaux, je cherche à établir une relation féconde entre le présent de l'œuvre et le passé de l'art.

Une relation ludique avec l'histoire de la musique prend forme à plusieurs niveaux simultanés. Jeux du souvenir et de l'oubli, en une interprétation-manipulation de la mémoire. Une reconstruction du passé où l'on expose, à travers l'œuvre, le procédé par lequel la mémoire fait, défait, refait son propre passé, en réévaluant constamment les statuts mouvants de l'histoire, en les projetant constamment dans nos visions de l'avenir, car la mémoire n'est pas neutre, elle produit le sens.

Cette attitude ne relève pas d'une forme de nostalgie. Il n'est pas question ici d'un retour à un paradis perdu de la musique. Il s'agit d'effacer les frontières entre le présent, le passé et l'avenir.

Rester au plus proche de Mozart tout en poussant encore plus loin ses propositions, ne serait-ce pas une forme tout à fait particulière de ce que Boulez appelait «l'écartèlement du visage »?

\section{Au bord du quai (ou de la forêt)...}

Au terme de sa réflexion théorique sur le pourquoi de son action, dans la conférence précitée, Lesage semble avoir d'abord et avant tout tenté de préserver cette liberté intérieure susceptible de lui permettre «l'exploration libre et 
hardie de l'imaginaire sonore ", dont Le projet Mozart est un éloquent témoignage. Sans vouloir tomber dans le relativisme bon teint, on peut souhaiter la même chose à tout créateur, que ses apprentissages, goûts, exclusions, positions le portent vers un courant ou l'autre: post-structuralisme, post-cagisme, post-minimalisme, post-spectralisme, post-xénakisme, post-utopisme, postrock-alternativisme, etc. C'est au creux de cette liberté (parfois perdue et retrouvée, jalousement préservée, quotidiennement et sereinement entretenue) que peuvent naître des œuvres susceptibles d'intéresser longtemps celle des auditeurs auxquels on s'adresse.

Bien qu'aujourd'hui le terme "accessibilité » soit le slogan d'une chasse aux sorcières élitistes, il faut pourtant dire qu'il y a dans Le projet Mozart une forme de clarté, de légèreté, d'humour que n'aurait pas dédaigné le «divin » inspirateur de cette pièce et qui lui confère une convivialité unique. Il s'agit d'une excellente mise en condition pour des promenades en des forêts plus obscures ou des croisières en des eaux plus agitées du projet Lesage. 